\title{
MOLUSCOS DE IMPORTÂNCIA ECONÔMICA NO BRASIL. II SUBULINIDAE, SUBULINA OCTONA (BRUGUIÉRE) (MOLLUSCA, GASTROPODA, PULMONATA, STYLOMMATOPHORA)
}

\author{
José Luiz de B. Araújo ${ }^{1}$ \\ Elizabeth Cristina de A. Bessa ${ }^{2}$
}

\begin{abstract}
MOLLUSCS OF ECONOMIC IMPORTANCE IN BRAZIL. II SUBULINIDAE, SUBULINA OCTONA (BRUGUIÉRE) (MOLLUSCA, GASTROPODA, PULMONATA, STYLOMMATOPHORA). A study of the anatomy of Subulina octona, mainly the genital and digestive systems, was undertaken in order to obtain additional data on the taxonomic position in the genus Subulina. The importance of the species as intermediate host for several helminths which occur in Brazil was also discussed.

KEY WORDS. Mollusca, Gastropoda, Pulmonata, Stylommatophora, Subulina octona
\end{abstract}

Dentre as várias espécies de moluscos já analisadas no Brasil, e comprovadas como hospedeiros intermediários de parasitos, principalmente em outros países do mundo, encontramos Subulina octona $(\mathrm{Br}$.), como uma das que em maior número de vezés é registrada como tal. Isto acontece, não só dentre os helmintos de animais, como também com relação a alguns do homem.

Foi referido como hospedeiro intermediário nos ciclos evolutivos de Platynosomum illiciens ( = fastosum Braun, 1901) do gato doméstico por MALDONADO (1945); Paratanaisia bragai ( = Tamerlania bragai) $($ Santos, 1934) de aves domésticas, por MALDONADO (1945); Postharmostomum gallinum Witenberg, 1923, de aves domésticas, por ALICATA (1940) e DUARTE (1977); Aelurostrongylus abstrusus (Railliet, 1898), do gato doméstico, por ALICATA (1964) e ASH (1962) e Davainea proglottina (Davaine, 1860), de aves por VAN VOLKENBERG (1937) in MALDONADO (1945). Algumas vezes citada como o único hospedeiro intermediário, outras vezes como um deles. A comprovação no Brasil foi feita com Postharmostomum gallinum (como segundo hospedeiro intermediário). Além do mais, consideramos um importante hospedeiro potencial para o Brasil, de uma parasitose exótica, causada pelo Angiostrongylus cantonensis, extremamente grave para o homem (já assinalada nas ilhas do Caribe), uma vez que, segundo MALEK \& CHENG (1974) e ALICATA (1964), S. octona também é capaz de atuar como hospedeiro intermediário deste nematódeo.

1) Departamento de Parasitologia Animal, Universidade Federal Rural do Rio de Janeiro, 23851-970 Itaguaí, Rio de Janeiro, Brasil. Bolsista da CAPES.

2) Bióloga. Bolsista do CNPq. 
Alguns autores brasileiros, como LANZIERI (1966), estudaram a espécie, no caso deste, em seus aspectos histológicos, sem contudo fazer comparação dos seus caracteres com os de outros gêneros da família comoLamelaxis, Opeas, Rumina, já referidos para o Brasil.

Levando-se em conta portanto, a importância das espécies de moluscos no estudo dos parasitos que delas necessitam para o desenvolvimento dos ciclos evolutivos, torna-se importante um bom conhecimento dessas espécies, não só do ponto de vista conquiliológico, como também da sua anatomia visceral, e, desta forma, melhor identificar os hospedeiros intermediários de muitos parasitos.

Subulina octona foi descrita por BRUGUIÉRE (1789), com base apenas nos caracteres conquiliológicos, apesar de CHEMNITZ (1786) ter feito sua descrição denominando-a ao mesmo tempo. Porém, tal descrição não foi considerada por não estar de acordo com as Regras Internacionais de Nomenclatura Zoológica. Outros autores fizeram redescrições morfológicas, principalmente dos caracteres conquiliológicos, mas, também de sua distribuíção geográfica e caracteres das partes moles, como: LAMARK (1822), FISCHER \& CROSSE (1878), VON MARTENS (1890/1901), TRYON \& PILSBRY (1906), THIELE (1931), MORRETES (1949), JAECKEL (1952), TILLIER (1959), LANZIERI (1966), BOFFI (1979), OLIVEIRA et al. (1981), BURCH (1960), bem como outros referindo-se às suas relações com parasitos como: MALDONADO (1945a, 1945b), ASH (1962), ROSEN et al. (1970), MALEK \& CHENG (1974), DUARTE (1977).

\section{MATERIAL E MÉTODOS}

O estudo foi conduzido nos laboratórios de Malacologia do Departamento de Parasitologia do Instituto de Biologia da Universidade Federal Rural do Rio de Janeiro, (UFRRJ), no Distrito de Seropédica, Itaguaí, Rio de Janeiro, para onde era transportado o material coletado.

\section{ORIGEM E MANUSEIO DO MATERIAL ESTUDADO}

Para o estudo morfológico dos moluscos, foram utilizados espécimens capturados em vários habitats terrestres nos estados do Rio de Janeiro (Valença, Rio das Flores, Três Rios, Paraíba do Sul, Itaguaí), de São Paulo (Santos e Piquete), do Rio Grande do Sul (Porto Alegre) e do Amapá (Macapá).

Os moluscos foram capturados nos locais de suas preferências (sob pedras, em madeira em decomposição, sobre plantas de jardim, em fendas junto a construções). Os espécimens capturados foram transportados para o laboratório em frascos de vidro com tampa perfurada que permitia uma pequena aeração. No laboratório, eram separados em lotes de acordo com a finalidade. Alguns eram destinados ao estabelecimento de colônias, sendo colocados em caixas onde se procurava reproduzir, tanto quanto possível, seu habitat. Tais moluscos eram utilizados em estudos morfológicos posteriores, observações bionômicas (alimentação, reprodução, etc.) ou infecções experimentais com 
parasitos para avaliação de sua susceptibilidade. Este material, quando fixado, era depositado na Coleção de Moluscos Hospedeiros Intermediários (Col. Mol. Hosp. Int.), no Departamento de Parasitologia Animal, do Instituto de Biologia da Universidade Federal Rural do Rio de Janeiro.

\section{ESTUDOS MORFOLÓGICOS}

Para os estudos morfológicos, o material, uma vez no laboratório, era dissecado, procurando-se isolar as partes mais importantes para determinação da espécie, no caso, a concha, o sistema digestivo (para a retirada da rádula e mandíbula) e o sistema genital.

Os espécimens vivos, foram colocados em água e aquecidos a $60^{\circ} \mathrm{C}$ para sacrifício, sendo retiradas as partes moles da concha. Outras vezes os moluscos eram dissecados em álcool glicerinado, quando se utilizava este líquido para sua conservação por ocasião da coleta. Quando, da dissecação, um detalhe da anatomia topográfica era julgado importante, executavam-se desenhos em câmara clara, acoplada ao microscópio estereoscópico.

Concluídas as dissecações e separadas as partes de interesse, procedia-se do seguinte modo: o bulbo da rádula era fervido em potassa a $10 \%$ para o isolamento de rádula e mandibula. As rádulas eram coradas em uma mistura de Vermelho Congo e Orange $\mathrm{G}$ após desidratação na série de álcoois e xilol. Em seguida, eram montadas em bálsamo-do-Canadá entre lâmina e lamínula. O sistema genital era desidratado na série de álcoois e posteriormente corado em carmin clorídrico, clarificando em creosoto, onde era conservado e estudado.

Para a distribuição geográfica da espécie, além das comprovações pessoais foram consultados: BOFFI (1979), DUARTE (1977), HAAS (1952), JAECKEL (1952), LANZIERI (1966), MORRETES (1949), OLIVEIRA et al. (1981), PILSBRY (1933), BURCH (1960).

\section{ESTUDOS BIONÔMICOS}

Algumas observações acerca do comportamento da espécie foram feitas nos seus habitats, onde se procurava acompanhar sua atividade em vários momentos, quando as circunstâncias se modificavam. Outras vezes, alguns dados foram obtidos em criadouros, onde os moluscos eram mantidos e observados. Estes criadouros constavam de caixas plásticas, fechadas com tampa telada para permitir aeração do ambiente. $O$ fundo era coberto por uma camada de terra, sempre rica em matéria orgânica sobre a qual se colocava pedras, pedaços de madeira em decomposição, sendo permanentemente mantido um certo grau de umidade. A alimentação dos moluscos constava de vegetais folhosos (principalmente alface) e de ração para aves levemente umedecida e colocada em pequenas placas de Petri. Visando conhecer as relações de algumas espécies com parasitos, foram feitos ensaios com o intuito apenas de se averiguar a susceptibilidade, quando era pesquisado o aparecimento de formas intermediárias dos parasitos utilizados. Estes ensaies constavam de infecções de moluscos com as formas infectantes dos parasitos em estudo. 


\section{RESULTADOS}

\section{Subulina octona (Bruguiére, 1789)}

Figs 1-11

Helix octona Indiae occidentalis Chemnitz, 1786:190, pl. 136, fig. 1264.

Bulimus octonus Bruguiére, 1789:325.

Bulimus octonus; Lamarck, 1822, 2a. part.: 124, fig. 27.

Achatina octona ; Orbigny, in La Sagra, 1853: 168, 169, pl. 11, figs 4-6.

Achatina octona; Reeve, 1849 . Achatina sp., 84 pl. XVII.

Subulina octona; Fisher e Crosse, 1878: 639, pl. XXV, figs 15 e 15a.

Subulina octona; Von Martens, 1890/1901: 298-300.

Subulina octona; Tryon e Pilsbry, 1906: 72-74, 222-224, pl. 12, figs 8-9; pl. 39, figs 28, 37, 39 e 40.

Subulina octona; Burch, J.B., 1960: 29, pl. VI, fig. C.

Subulina octona Haas, 1962: 49-52, pl. VIII, figs A-F.

Subulina octona; Lanzieri, 1966: 1-29, 32-44, figs 2-13.

Concha (Figs 1-2). É pequena, medindo aproximadamente $13 \times 4 \mathrm{~mm}$, com a espira muito longa, graças ao número de voltas que apresenta (oito). A amplitude das voltas aumenta progressivamente até a volta corporal. Não apresenta nenhuma ornamentação característica, evidenciando de forma não muito marcada, as linhas de crescimento. A abertura é de forma ovalada sem dentes ou lamelas e com o peristoma cortante. $\mathrm{O}$ bordo columelar apresenta uma leve projeção alongada no sentido do eixo, mais alargada em sua metade. As suturas são evidentes, porém não muito profundas.

Rádula (Fig. 3). É uma lâmina muito delgada, com 20 dentes de cada lado, mais desenvolvidos e mostrando particularidades apreciáveis somente até a quinta fileira. Daí em diante os dentes são muito pequenos e reduzem-se progressivamente até a margem, onde são rudimentares. A margem anterior do dente recobre a posterior do dente anterior da mesma fileira. O dente central é muito reduzido e sem características marcantes. Tem a forma retangular, com uma leve protuberância central, no mesmo sentido do eixo maior do dente. Pelas pequenas dimensões deste dente, a fileira central mostra-se como uma estreita linha em toda a extensão da rádula. O primeiro dente é quase simétrico, apenas com o ângulo posterior externo mais agudo. A cúspice central é desenvolvida e com duas cúspides menores colocadas interna e externamente a ela. Estas características se repetem até o quarto dente. O quinto dente é um pouco menor e provido de duas pequenas cúspides. Do sexto dente até a margem, os dentes não tem características marcantes, aparecendo então discretamente, nos primeiros que seguem, um espessamento um pouco maior da margem anterior.

Mandíbula (Fig. 4). É do tipo odontognato, com placas de forma aproximadamente retangular, cujos bordos anteriores são levemente arredondados e mais estreitos que o restante do corpo da mandíbula. A metade anterior tem os bordos laterais marcados, articulando-se com as placas contíguas, porém com muito pouca quitinização, o que lhe dá a flacidez necessária para a mobilidade.

Ovos (Figs 5, 6). São esféricos, de cor esbranquiçada, medindo aproximadamente $2 \mathrm{~mm} \times 1,6 \mathrm{~mm}$, devido aos dois achatamentos paralelos que possuem. 


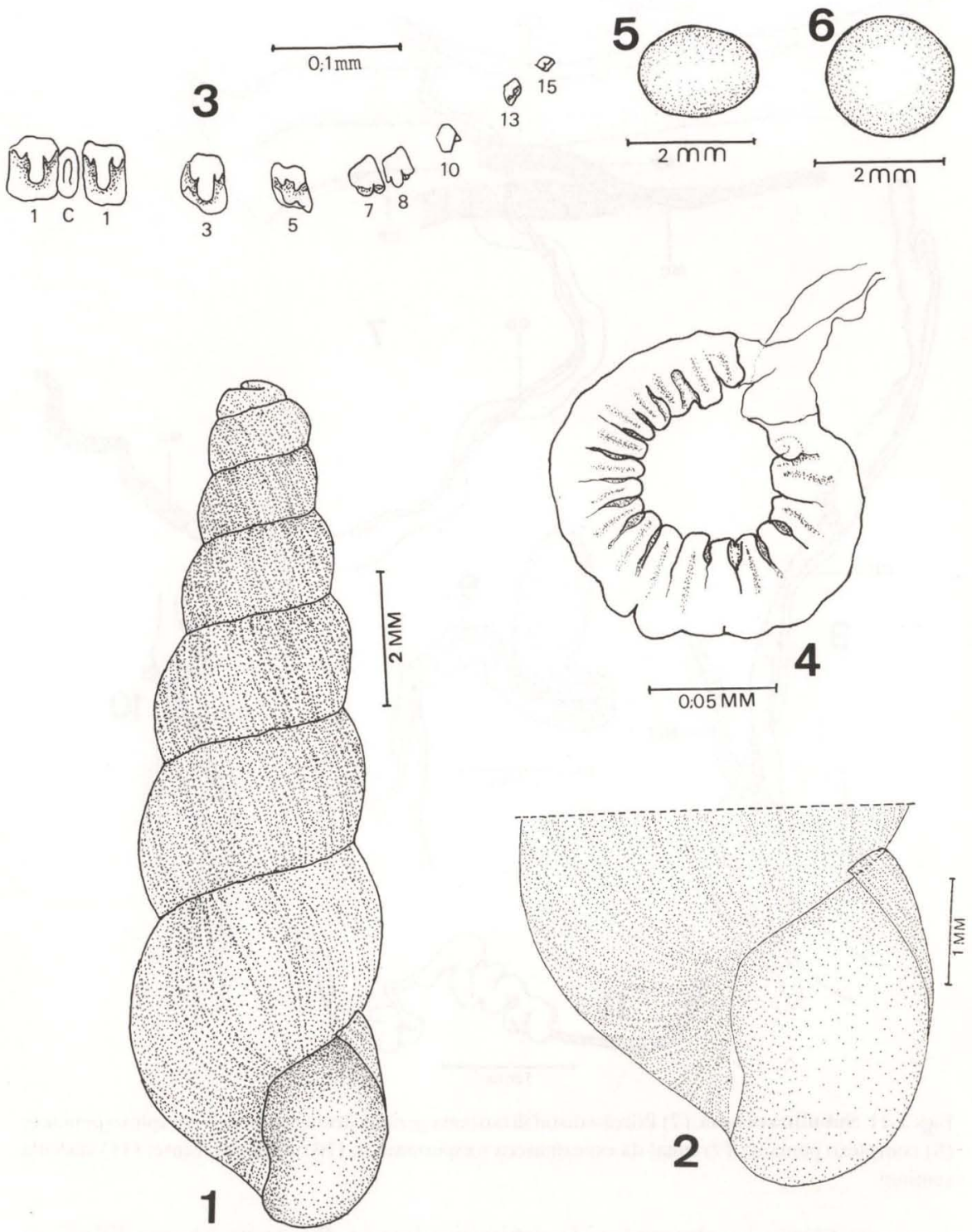

Figs 1-6. Subulina octona. (1) Concha; (2) abertura da concha; (3) rádula; (4) mandibula; (5-6) ovo.

Sistema genital (Fig. 7). Inicia-se pelo ovotestis aproximadamente a partir da terceira volta da espira, sendo em seu conjunto, um sistema bastante longo, decorrente da forma da concha e ocupando sempre uma posição próxima do eixo columelar. $\mathrm{O}$ ovotestis é formado por um pequeno grupo de folículos pouco individualizados. Seus canais vão ter ao canal coletor do ovotestis (Fig. 11) de extensão mais ou menos retilínea, apoiado na glândula de albumina e 


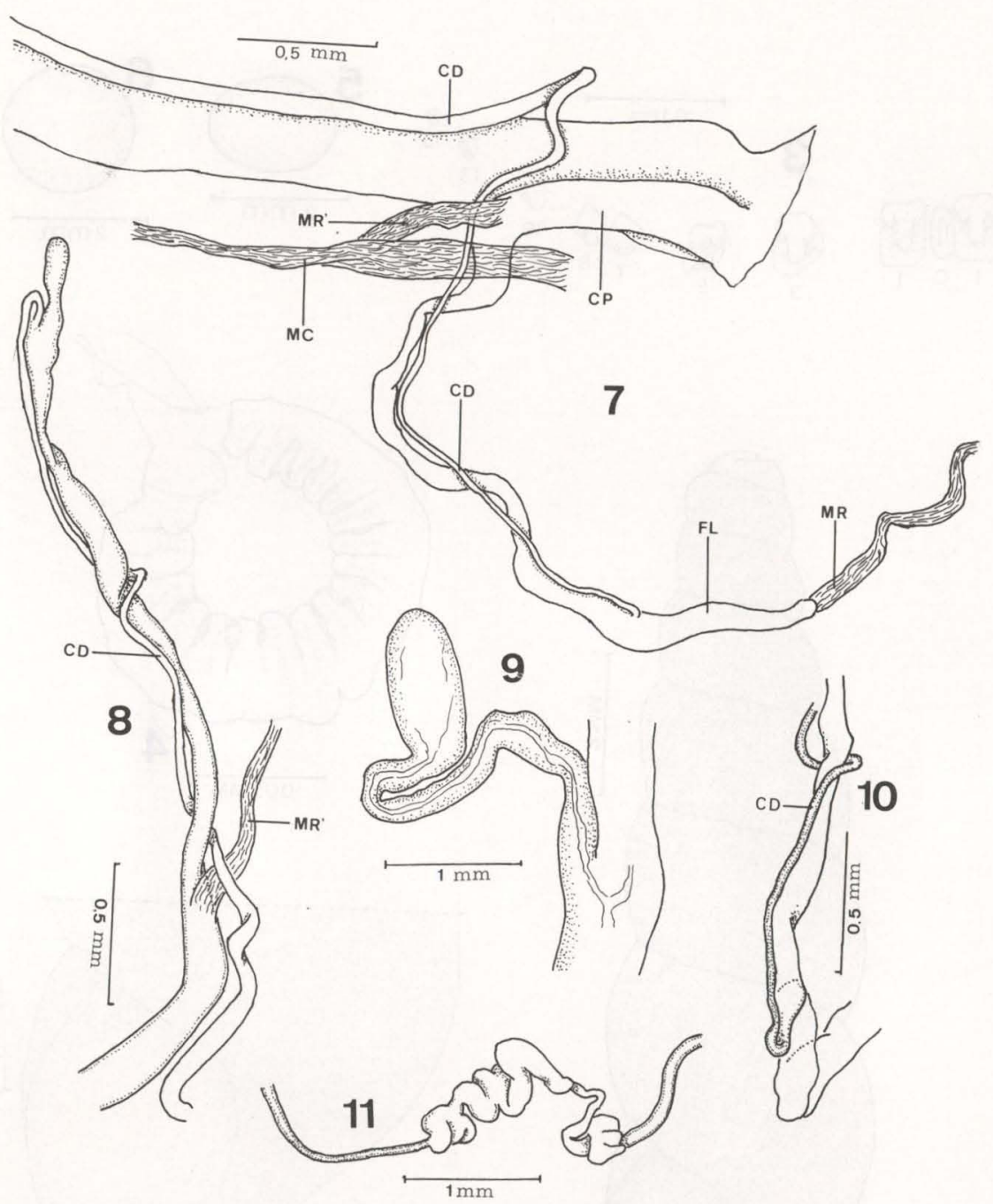

Figs 7-11. Subulina octona. (7) Porção distal do sistema genital, destacando-se o complexo peniano; (8) complexo peniano; (9) canal da espermateca e espermateca; (10) canal deferente; (11) vesícula seminal.

fracamente preso a ela por tecido conjuntivo frouxo. Bruscamente modifica sua forma, tornando-se um tubo fortemente enrolado, de calibre maior, sem contudo, modificar sua direção, e apresentando importantes diferenças histológicas das regiões vizinhas (LANZIERI, 1966). O canal hemafrodita tem aspecto semelhante ao canal coletor do ovotestis, porém é mais curto e ligeiramente mais espesso. Sua desembocadura se faz no receptáculo seminal, que se coloca na face côncava da glândula de albumina um pouco mais próximo da extremidade proximal desta. 
Glândula de albumina. De forma achatada acompanhando a volta da espira e em grande parte colocando-se entre esta e a glândula digestiva, variando sua forma, dimensões e aspecto geral de acordo com a atividade reprodutiva do animal. $\mathrm{O}$ ovispermoduto, é também um órgão alongado decorrente da forma geral do molusco. Contudo, em face de sempre, em exemplares adultos, serem encontrados ovos "in utero" o órgão destende-se muito, modificando-se a posição do pregueamento uterino, tornando-o um pouco mais baixo, bem como modificando a próstata, em um órgão laminar, com aparência mais curta que o útero. O oviduto (Fig. 17) é longo, tendo o canal deferente (Figs 17, 18, 20) preso em grande parte de sua extensão, por tecido conjuntivo frouxo. Este canal abandona este trajeto a alguma distância do átrio genital, para adotar a mesma situação junto ao complexo peniano. Desemboca no complexo peniano, sem demonstrar externamente modificações importantes.

Canal do receptáculo seminal (Fig. 9). É um curto e calibroso canal que tem sua origem bastante distante do átrio genital, deixando com isso o duto vaginal longo. Sua luz é estreita em toda sua extensão, mesmo nas proximidades do receptáculo seminal, onde se reduz o calibre do canal. Apoia-se ao longo do ovispermoduto, ficando o receptáculo seminal sobre as últimas dobras do útero, nas proximidades da origem do canal deferente.

Complexo peniano (Figs 7, 8, 10). Muito longo e delgado, tendo esta característica na maior parte de sua vida, com a diferença bem maracada para as demais partes do sistema genital. A região fálica representa a maior parte do órgão, isto porque, nas preparações efetuadas não demonstrou diferenças importantes que a diferenciariam da região epifálica, se existisse esta, como por exemplo a presença do tecido glandular. Não possue bainha muscular envolvendo-a. $\mathrm{O}$ que se nota de forma mais evidente, é um maior calibre até o ponto onde se insere uma faixa muscular originada de uma faixa do músculo columelar. O músculo retrator do pênis tem inserção terminal no flagelo e é relativamente longo. $\mathrm{O}$ flagelo é curto, terminando em fundo cego, possue uma luz não muito evidente nas preparações habituais. Seu calibre pode se apresentar diferente em relação ao falus, conforme o estado fisiológico do órgão no momento.

Distribuição geográfica. BRASIL: Amapá, Amazonas, Pará, Ceará, Bahia, Rio de Janeiro, Minas Gerais, São Paulo, Paraná, Rio Grande do Sul, Rondônia.

\section{REFERÊNCIAS BIBLIOGRÁFICAS}

ASH, L.R. 1962. Helminth parasites of dogs and cats in Hawaii. J. Parasitol. 48: 63-65.

ALICATA, J.E. 1940. The life cicle of Postharmostomum gallinum the cecal fluke of poultry. J. Parasitol. 26 (2): 135-143.

.1964. Parasitic infections of man and animals in Hawaii. Agricultural Experiment Station University of Hawaii Tech. Bul. 61: 1-138.

BOFFI, A.V. 1979. Moluscos brasileiros de interesse médico e econômico. Rio de Janeiro, Hucitec Ed., 376p.

BRUGUIÉRE, J.G. 1789/1792. Encyclopedie Méthodique. Historie Naturelle 
des Vers. Paris et Liége. Tomo I part. 1 (1789): 1-344. Tomo II part. 2 (1792): 345-758.

CHEMNITZ, J.H. 1786.In: F.H.W. MARTINI \& J.H. CHEMNITZ (ed.). Neus

Systematisches Conchylien Cabinet. Nurenberg, XXVI + 194p.

BURCH, J.B. 1960. Some snails and slugs of Quarantine significance to the United States. Sterliana 2: 13-53.

DUARTE, M.J.F. 1977. O ciclo evolutivo de Postharmostomum gallinum Witenberg, 1923, no Estado do Rio de Janeiro. Tese, não publicada, Universidade Federal Rural do Rio de Janeiro, 69p.

FISHER, P. \& H. CROSSE. 1878. Mission Scientifique au Mexique et dans

l'Amérique Centrale. Recherches zoologiques: p.153-699.

HAAS, F. 1962. Caribbean Land Molluscs: Subulinidae and Oleacinidae, Studies on the fauna of Curaçao and other Caribbean Islands. XIII (58): 49-60. The Hague Martius Nijhoff.

LAMARK, J.B.P.A. 1819/1822. Histoire Naturelle des Animaux sans vertebres 6 (1) (1819), IV +343p.; (2) (1822), 232p. Paris.

LANZIERI, P.D. 1966. Alguns aspectos morfo-estruturais do aparelho genital de Subulina octona (Bruguière, 1792) (Gastropoda, Pulmonata, Subulinidae). Tese, não publicada, $44 \mathrm{p}$.

LA SAGRA, M.R. DE. 1853. Histoire Physique, Politique et Naturelle de l' Ile de Cuba. Mollusques (par Alcide D’ Orbigny). Tomo I (1841): 1-208; Tomo II (1842): 209-264.

MALDONADO, J.F. 1945a, The life cicle of Tamerlania bragai Santos, 1934 (Eucotylidae) a kidney fluke of domestic pigeons. J. Parasitol. 31 (5): 306-314.

. 1945b. Ciclo vital e biologia del Platynosomum fastosum Kossak, 1910 (Trematoda: Dicrocoelidae). J. Publ. Hlth Trop. Med. 20 (17): 34-60.

MALEK, E.A. \& T.C. CHENG. 1974. Medical and Economic Malacology. Academic Press Ed. X+398p.

MORRETES, F.L. 1949. Ensaio de Catálogo dos Moluscos do Brasil. Arq. Mus. Par. 7: 5-216.

OLIVEIRA, M.P.; G.J.R. REZENDE \& G.A. CASTRO. 1981. Catálogo dos

Moluscos da Universidade Federal de Juiz de Fora. Juiz de Fora, Universidade Federal de Juiz de Fora Ed., 520p.

PILSBRY, H.A. 1933. Zoological results of the Matto Grosso expedition to Brazil in 1931. II. Mollusca. Proc. Acad. Nat. Sci. Philad. 85: 67-76.

REEVE, L.A. 1849. Conchologia Iconica. Illustrations of the shells of molluscous animals. Vol. V.

ROSEN, L.; L.A. ASH \& G.D. WALLACE. 1970. Life history of the canine lungworm Angyostrongylus vasorum (Baillet). Am J.Vet. Res. 31 (1): 131-143. THIELE, J. 1931. Handbuch der Systematischen Weichtierkunde 1: VI+ 1778p, Jena.

TILLIER, S. 1989. Comparative Morphology, phylogeny and classification of land snails and slugs (Gastropoda: Pulmonata: Stylommatophora). Mala- 
cologia 30 (1-2): 1-303.

TRYON, G.W. \& PILSBRY, H.A. 1906. Manual of Conchology. XVIII: VII + $357 \mathrm{p}$.

VON MARTENS, E. 1890/1901. Biologia Centrali-Americana. Land and Freshwater mollusca. XXVIII + 705p.

Recebido em 31.XII.1991; aceito em 25.XI. 1993. 